\title{
Receptor-like kinases take center stage in plant biology
}

\author{
Jian-Min Zhou ${ }^{1 *} \&$ Wei-Cai Yang ${ }^{2 * *}$ \\ ${ }^{1}$ State Key Laboratory of Plant Genomics, Institute of Genetics and Developmental Biology, Chinese Academy of Sciences, Beijing 100101, \\ China \\ ${ }^{2}$ State Key Laboratory of Molecular Development, Institute of Genetics and Developmental Biology, Chinese Academy of Sciences, Beijing \\ 100101, China
}

Citation: Zhou, J.M., and Yang, W.C. (2016). Receptor-like kinases take center stage in plant biology. Sci China Life Sci 59, 863-866. doi: $10.1007 / \mathrm{s} 11427-016-5112-8$

Like the animals, higher plants must perceive and interpret numerous external and endogenous cues to properly program growth and development and physiological responses. Animal cells use diverse receptors to perceive external signals to regulate cellular processes. Thus, receptor-tyrosine kinases (RTKs) are responsible for perceiving growth regulators, Toll-Like Receptors (TLRs) are responsible for detecting microbial molecular patterns to activate innate immunity, whereas G protein-coupled receptor (GPCR) superfamily are responsible for interpreting numerous chemical signals such as odors. Plant genomes, however, do not encode RTKs, TLRs, nor GPCRs. Instead, the plasmamembrane of plant cells is occupied by a superfamily of receptor-like kinases (RLKs; Shiu and Bleecker, 2003). Unlike RTKs, plant RLKs are exclusively serine/threonine kinases according to phylogeny of protein kinases, although tyrosine phosphorylation does occur to RLKs. RLKs can act as receptors, co-receptors, and other regulatory partners of receptor kinases, and play a central role in the maintenance of stem cell, fertilization, growth and development, adaptation to biotic and abiotic stresses, and establishing symbiotic relationship with beneficial microbial organisms. Thus the single plant RLK superfamily is functionally equivalent to animal RTKs, TLRs, and GPCRs. Understanding how RLKs act in these processes has been at the forefront of plant biology.

*Corresponding author (email: jmzhou@genetics.ac.cn)

**Corresponding author (email: wcyang@genetics.ac.cn)
RLRKs typically contain a highly variable ectodomain, a single pass transmembrane domain, and a cytoplasmic kinase domain. The ectodomains include leucine-rich repeat (LRR), Lysin motif (LysM), lectin, etc. that are expected to recognize different ligands. RLKs known to act as receptors are called receptor kinases (RKs). Some of the RLK superfamily members do not contain a transmembrane domain and ectodomain, and these are called receptor-like cytoplasmic kinases (RLCKs). Accumulating evidence indicates that RLCKs act together with RKs in receptor complexes (Tang et al., 2008; Zhang et al., 2010). In addition, plants also encode a large number of proteins containing a single transmembrane domain and an ectodomain, but no kinase domain. These proteins are called receptor-like proteins (RLPs), some of which have been shown to act as receptor proteins (RPs) and transduce signals by interacting with RLKs. In this issue, there are three review articles and one research article addressing regulatory mechanism and role of RLKs and RLPs in plant reproduction, immunity, and growth and development, giving readers a flavor of diverse research fields of RLK-mediated signaling and illustrating the importance of RLKs in plant biology.

Sexual reproduction involving male and female gametophytes is key to success of flowering plants. It not only increases genetic diversity and accelerates evolution of plant species, but also provides the majority of foods to mankind. The pollen grain containing sperms germinates upon landing on stigma, forming a pollen tube that penetrates various female tissues to reach synergids and egg cells within the 
ovule, resulting in the rupture of pollen tube and release of sperm for double fertilization. A large number of flowering plant species display self-incompatibility, a phenomenon in which stigma rejects pollen grains of the same genotype to ensure out-crossing. This incompatibility is triggered upon the recognition of pollen grains by the stigma of the same haplotype, highlighting the first male-female communication (Nasrallah and Nasrallah, 2014). For compatible pollen grains that successfully germinate on stigma, the pollen tube assume polarized growth in pistil, style and transmitting track. In this process, the female-derived signals promote pollen tube growth and integrity. In the last stages of growth, the pollen tube penetrates septum and moves accurately toward an ovule, enters the micropylar opening of embryo sac where the fertilization occurs. During these stages, pollen tube growth is guided by peptide signals from ovule and synergid cells, a process called pollen tube guidance, which has been a major focus in the field of fertilization (Higashiyama and Takeuchi, 2015; Takeuchi and Higashiyama, 2015; Wang et al., 2016b). Finally, additional communication takes place between pollen tube and synergid cells upon the arrival of pollen tube in the embryo sac to stimulate proper rupture of the pollen tube. RLKs localized in female or male cells play a central role in the perception and interpretation of signals from the opposite sex at all stages of fertilization. The review by $\mathrm{Li}$ and Yang ( $\mathrm{Li}$ and Yang, 2016) provides an excellent coverage on how RLKs mediate the sophisticated male-female communications during each stage.

The review by $\mathrm{Li}$ et al. (Li et al., 2016) provides an account of recent advances in the understanding of roles and mechanisms by which RLKs and RLPs regulate plant disease resistance. Although plants do not possess an adaptive immune system that operates in vertebrates, they possess a powerful innate immune system highly analogous to that in animals. Plant innate immune system relies on the perception of microbial infection by plasmamembrane-localized immune receptors and cytoplasmic immune receptors. The former is composed of RKs and RPs collectively called pattern-recognition receptors (PRRs) that detect molecular patterns of host or microbe origin during infection, and activate intracellular signaling processes to trigger a variety of defenses. Pattern-triggered immunity (PTI) has been a major topic in the field of plant-microbe interactions, and known PRRs are rapidly expanding as a result of active research on new microbial patterns. Accumulating evidence indicates ligand-induced oligomerization of PRRs with co-receptors as a general mechanism in the activation of PRRs (Liu et al., 2012; Sun et al., 2013a). Recent studies have also supported BIK1 and related RLCKs as a major link between PRRs and downstream responses (Zhang et al., 2010; Kadota et al., 2014; Li et al., 2014). Work in recent years have also uncovered positive and negative regulatory mechanisms controlling the PRR complex, which are critical for a tight control of plant immunity that prevents detrimental activation of immunity in the absence of infection. Li et al. (Li et al., 2016) summarize well-known and newly identified PRRs, their respective ligands/patters, and composition and dynamic regulation of PRR complexes.

The review article by Fan et al. (Fan et al., 2016) focuses on a small family of RLKs called SERKs, so named because the founding member was first identified as an RLK involved in somatic embryo biogenesis (Schmidt et al., 1997). There are only five members of SERKs in Arabidopsis, each containing a small LRR domain. The first line of evidence that SERKs may act as a co-receptor for a LRR-RK comes from two reports showing that SERK3 is a BRI1-associated kinase (BAK1) that functions in brassinosteroid (BR) signaling (Nam and Li, 2002; Li et al., 2002). Structural studies of the BRI1-BR-BAK1 complex demonstrated that BAK1 indeed is the co-receptor for BRs (Santiago et al., 2013; Sun et al., 2013b). Subsequent analyses showed that additional members of SERK family also act additively in BR signaling. Genetic and protein-protein interaction studies have since showed that members of SERK family act together with an increasing number of LRR-LRK to perceive molecular patterns associated with infection and peptide hormones to regulate immunity, growth and development, and cell differentiation. Fan et al. (Fan et al., 2016) provide an updated account of diverse LRR-RLK-mediated signaling pathways in which SERKs act as co-receptors and in-depth analyses of underlying mechanism using existing data.

The research article by Wang et al. (Wang et al., 2016a) investigates how the phytopathogenic bacterium Xanthomonas oryzae oryzae suppresses plant innate immunity during infection. Xoo is a gram-negative bacterium and a causal agent of rice bacterial blight. It secretes a repertoire of effector proteins into the host cell for parasitism. The authors investigate role of one such effector called XopR in the inhibition of pattern-triggered immunity. Heterologous expression in Arabidopsis showed that XopR localizes to plasmamembrane. GST pull-down and co-IP assays indicated that XopR can interact with BIK1. Consistent with these findings, transgenic expression of XopR in Arabidopsis plants inhibited FLS2-mediated stomatal closure in response to bacterial molecular pattern flg22, indicating that XopR can inhibit PTI by targeting BIK1. Thus XopR joins several other bacterial effectors as PTI-inhibitors by targeting BIK1 and BIK1-related RLCKs (Zhang et al., 2010; Feng et al., 2012; Feng and Zhou, 2012). In the future it will be important to test whether XopR uses a similar mechanism to inhibit PTI in rice and elucidate the underlying biochemical mechanism. 
Only a small number of the $\sim 600$ plant RLKs have been assigned for biological functions. What are the roles of the remaining RLKs? How many of these RLKs are receptors? What are their ligands? How different RKs regulate diverse biological processes? Future research answering these questions will continue to be a center piece in plant biology.

Compliance and ethics The author(s) declare that they have no conflict of interest.

Fan, M., Wang, M., and Bai, M.Y. (2016). Diverse roles of SERK family genes in plant growth, development and defense response. Sci China Life Sci 59, 889-896.

Feng, F., and Zhou, J.M. (2012). Plant-bacterial pathogen interactions mediated by type III effectors. Curr Opin Plant Biol 15, 469-476.

Feng, F., Yang, F., Rong, W., Wu, X., Zhang, J., Chen, S., He, C., and Zhou, J.M. (2012). A Xanthomonas uridine 5'-monophosphate transferase inhibits plant immune kinases. Nature 485, 114-118.

Higashiyama, T., and Takeuchi, H. (2015). The mechanism and key molecules involved in pollen tube guidance. Annu Rev Plant Biol 66, 393-413.

Kadota, Y., Sklenar, J., Derbyshire, P., Stransfeld, L., Asai, S., Ntoukakis, V., Jones, J.D., Shirasu, K., Menke, F., Jones, A., and Zipfel, C. (2014). Direct regulation of the NADPH oxidase RBOHD by the PRR-associated kinase BIK1 during plant immunity. Mol Cell 54, 43-55.

Li, H., and Yang, W. (2016). RLKs orchestrate the signaling in plant male-female interaction. Sci China Life Sci 59, 867-877.

Li, J., Wen, J., Lease, K.A., Doke, J.T., Tax, F.E., and Walker, J.C. (2002). BAK1, an Arabidopsis LRR receptor-like protein kinase, interacts with BRI1 and modulates brassinosteroid signaling. Cell 110, 213-222.

Li, L., Li, M., Yu, L., Zhou, Z., Liang, X., Liu, Z., Cai, G., Gao, L., Zhang, X., Wang, Y., Chen, S., and Zhou, J.M. (2014). The FLS2-associated kinase BIK1 directly phosphorylates the NADPH oxidase RbohD to control plant immunity. Cell Host Microbe 15, 329-338.

Li, L., Zhou, Z., and Zhou, J.M. (2016). Plant pattern-recognition receptors controlling innate immunity. Sci China Life Sci 59, 878-888.
Liu, T., Liu, Z., Song, C., Hu, Y., Han, Z., She, J., Fan, F., Wang, J., Jin, C., Chang, J., Zhou, J.M., and Chai, J. (2012). Chitin-induced dimerization activates a plant immune receptor. Science 336, 1160-1164.

Nam, K.H., and Li, J. (2002). BRI1/BAK1, a receptor kinase pair mediating brassinosteroid signaling. Cell 110, 203-212.

Nasrallah, J.B., and Nasrallah, M.E. (2014). S-locus receptor kinase signalling. Biochem Soc Trans 42, 313-319.

Santiago, J., Henzler, C., and Hothorn, M. (2013). Molecular mechanism for plant steroid receptor activation by somatic embryogenesis Co-receptor kinases. Science 341, 889-892.

Schmidt, E.D., Guzzo, F., Toonen, M.A., and de Vries, S.C. (1997). A leucine-rich repeat containing receptor-like kinase marks somatic plant cells competent to form embryos. Development 124, 2049-2062.

Sun, Y., Li, L., Macho, A.P., Han, Z., Hu, Z., Zipfel, C., Zhou, J.M., and Chai, J. (2013a). Structural basis for flg22-induced activation of the Arabidopsis FLS2-BAK1 immune complex. Science 342, 624-628.

Sun, Y., Han, Z., Tang, J., Hu, Z., Chai, C., Zhou, B., and Chai, J. (2013b). Structure reveals that BAK1 as a co-receptor recognizes the BRI1-bound brassinolide. Cell Res 23, 1326-1329.

Takeuchi, H., and Higashiyama, T. (2016). Tip-localized receptors control pollen tube growth and LURE sensing in Arabidopsis. Nature 531, 245-248.

Tang, W., Kim, T.W., Oses-Prieto, J.A., Sun, Y., Deng, Z., Zhu, S., Wang, R., Burlingame, A.L., Wang, Z.Y. (2008). BSKs mediate signal transduction from the receptor kinase BRI1 in Arabidopsis. Science 321, 557-560.

Wang, S., Sun, J., Fan, F., Tan, Z., Zou, Y., and Lu, D. (2016a). A Xanthomonas oryzae pv. Oryzae effector, XopR, associates with receptor-like cytoplasmic kinases and suppress PAMP-triggered stomatal closure. Sci China Life Sci 59, 897-905.

Wang, T., Liang, L., Xue, Y., Jia, P.F., Chen, W., Zhang, M.X., Wang, Y.C., Li, H.J., and Yang, W.C. (2016b). A receptor heteromer mediates the male perception of female attractants in plants. Nature 531, 241-244.

Yang, W.C., Shi, D.Q., and Chen, Y.H. (2010). Female gametophyte development in flowering plants. Annu Rev Plant Biol 61, 89-108.

Zhang, J., Li, W., Xiang, T., Liu, Z., Laluk, K., Ding, X., Zou, Y., Gao, M., Zhang, X., Chen, S., Mengiste, T., Zhang, Y., and Zhou, J.M. (2010). Receptor-like cytoplasmic kinases integrate signaling from multiple plant immune receptors and are targeted by a Pseudomonas syringae effector. Cell Host Microbe 7, 290-301. 


\section{Biographical Sketches}

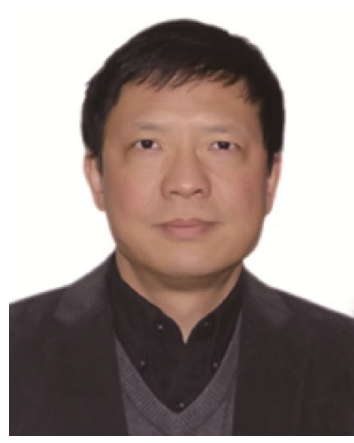

Jian-Min Zhou is known for his research on plant-microbe interactions. He was born in Chengdu in February 1964. He serves on the board of International Society for Molecular Plant-Microbe Interactions and international journals including PLoS Pathogens, eLife, Molecular Plant, and Current Protocols in Plant Biology. His research mainly focuses on plant immunity and bacterial pathogensis.

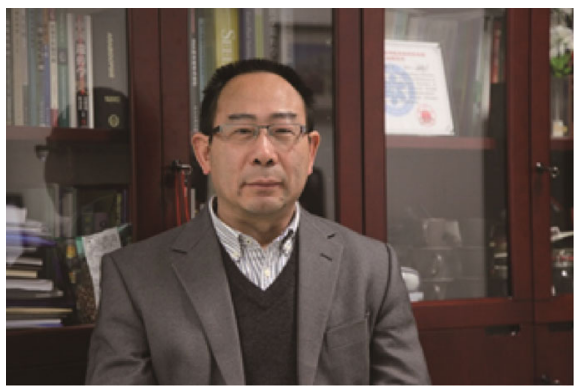

Wei-Cai Yang is a renowned plant biologist in China. He was born in Chongqing in February 1964. He is the director of the Institute of Genetics and Developmental Biology, Chinese Academy of Sciences and director of the Chinese State Key Laboratory of Molecular Development. His research emphasizes on molecular and cellular mechanisms underlying reproduction in flowering plants.

Open Access This article is distributed under the terms of the Creative Commons Attribution License which permits any use, distribution, and reproduction in any medium, provided the original author(s) and source are credited. 